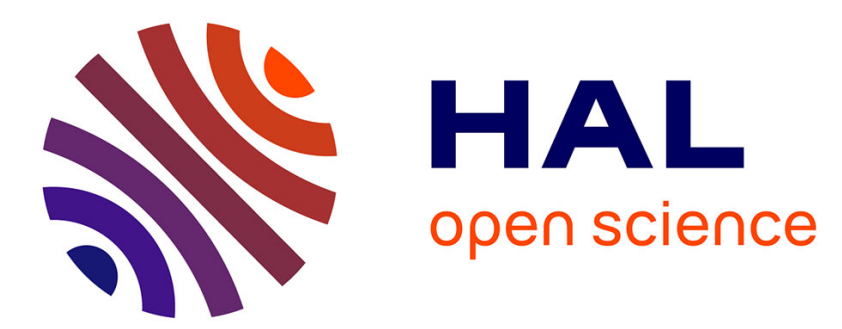

\title{
Bordeaux et la peste dans la première moitié du XVIIe siècle \\ Laurent Coste
}

\section{To cite this version:}

Laurent Coste. Bordeaux et la peste dans la première moitié du XVIIe siècle. Annales du Midi : revue archéologique, historique et philologique de la France méridionale, 1998, 110, pp.457 - 480. 10.3406/anami.1998.2599 . hal-02098242

\section{HAL Id: hal-02098242 \\ https://hal.science/hal-02098242}

Submitted on 4 May 2020

HAL is a multi-disciplinary open access archive for the deposit and dissemination of scientific research documents, whether they are published or not. The documents may come from teaching and research institutions in France or abroad, or from public or private research centers.
L'archive ouverte pluridisciplinaire HAL, est destinée au dépôt et à la diffusion de documents scientifiques de niveau recherche, publiés ou non, émanant des établissements d'enseignement et de recherche français ou étrangers, des laboratoires publics ou privés. 


\section{Bordeaux et la peste dans la première moitié du XVIle siècle} Laurent Coste

\section{Citer ce document / Cite this document :}

Coste Laurent. Bordeaux et la peste dans la première moitié du XVIle siècle. In: Annales du Midi : revue archéologique, historique et philologique de la France méridionale, Tome 110, №224, 1998. pp. 457-480;

doi : https://doi.org/10.3406/anami.1998.2599

https://www.persee.fr/doc/anami_0003-4398_1998_num_110_224_2599

Fichier pdf généré le 18/04/2018 


\section{Zusammenfassung}

Bordeaux und die Pest in der ersten Hälfte des 17. Jahrhunderts.

Während der ersten Hälfte des 17. Jahrhunderts, und besonders in den Jahren 1604 und 1629, wurde Bordeaux, wie auch viele andere Städte, von Pestepidemien verheert. Die örtlichen Obrigkeiten, und hier vor allem die bordelaiser Stadtverwaltung sowie der Oberste Provinzialgerichthof, taten alles in ihrer Macht stehende, um dieser Heimsuchung entgegen zu treten. Der unter der Leitung einer Gesundheitsbehörde geführte Kampf gegen die Pest (Absonderung, Krankenhauseinweisung, medizinische Fürsorge und Bittgottesdienste) erwies sich jedoch, gemessen an den von der Stadt aufgewandten erheblichen Geldmitteln, als wenig erfolgreich.

\section{Résumé}

Au cours de la première moitié du XVIle siècle, et plus particulièrement en 1604 et 1629, Bordeaux fut, comme beaucoup d'autres villes, touchée par des épidémies de peste. Les autorités locales, avec au premier rang la jurade et le parlement, s'efforcèrent de contenir ce fléau. Mais la lutte engagée sous le contrôle d'un Bureau de la santé (quarantaine, hospitalisation, soins médicaux et prières) se révéla peu efficace, eu égard à l'importance des sommes dépensées par la ville.

\section{Abstract}

Bordeaux and the Plague in the First Half of the Seveteenth-Century.

During the first half of the seventeenth-century, and in particular between 1604 and 1629, Bordeaux was, like many other cities, hit by plague epidemics. Local authorities, led by the jurade and the parlement, struggled to contain this calamity. But the battle thus entered into, under the control of a Health Office (quarantine, hospitalization, medical care and prayers) was revealed to be not very efficient given the importance of the sums of money spent by the city. 


\section{Laurent COSTE*}

\section{BORDEAUX ET LA PESTE DANS LA PREMIÈRE MOITIÉ DU XVII ${ }^{\mathrm{e}}$ SIĖCLE}

«La peste augmente de telle façon en ceste ville qu'il n'y a personne qui aye moyen de vivre ailleurs qui ne l'ayt abandonné ", écrivait le maréchal de Matignon à Henri III le 30 juin 1585'. L'épidémie qui frappait Bordeaux en cette fin de XVI ${ }^{\mathrm{e}}$ siècle est avant tout connue pour l'attitude fort prudente de son maire, Michel de Montaigne, qui préféra rester à l'écart de la ville². Mais, avant la lente rémission de la seconde moitié du XVII ${ }^{e}$ siècle, la principale ville de Guyenne fut confrontée à plusieurs reprises au fléau épidémique sous les règnes de Henri IV et de Louis XIII. Outre les petites alertes, la maladie frappa très durement en 1604-1605 et en 1629-1631. Malgré l'état lacunaire des registres paroissiaux conservés et les destructions causées par l'incendie des Archives municipales des 13-14 juin 1862 aux registres de délibérations de la jurade et du Bureau de la santé, la situation bordelaise, assez peu étudiée jusqu'ici ${ }^{3}$, paraît semblable à celle des autres grandes villes du royaume, comme Lyon, Amiens ou Clermont-Ferrand ${ }^{4}$.

Après deux décennies de latence, la maladie contagieuse réapparut donc à Bordeaux au début du XVII e siècle puis un quart de siècle plus tard, ses méfaits étant alors accentués par une conjoncture particulièrement difficile avec la

* 121 , route de Fargues, 33360 Carignan-de-Bordeaux.

1. Archives historiques de la Gironde, Bordeaux, 1873, t. 14, n 197, p. 290.

2. «Vaut-il que je me hasarde d'aller en la ville, vu le mauvais état en quoi elle est, notamment pour des gens qui viennent d'un si bon air comme je fais », écrivait-il aux jurats restés sur place. Cité par J. PlatTard, Montaigne et son temps, Paris, 1933, p. 246.

3. L'ouvrage de G. PÉRY, Les épidémies de Bordeaux pendant les $15^{e}$, $16^{e}$ et $17^{e}$ siècles, Bordeaux, Gounouilhou, 1867, est surtout anecdotique. $C f$. J.-N. BIRABEN, Les hommes et la peste en France et dans les pays européens et méditerranéens, Paris, 1976, deux volumes.

4. M. LuCENET, Lyon malade de la peste, Lyon, 1981 ; M. MALPART, La peste à Amiens au $17^{e}$ siècle. Étude médico-historique, Amiens, 1938 ; P. CHARBONNIER, «La peste de 1631 à Clermont », Revue d'Auvergne, 1965, t. 79, p. 97-127.

Docteur en histoire, Laurent Coste est maître de conférences d'histoire moderne à l'Université Michel-de-Montaigne de Bordeaux. Il travaille actuellement sur l'administration municipale de Bordeaux aux XVII ${ }^{\mathrm{e}}$ et $\mathrm{XVIII}{ }^{\mathrm{e}}$ siècles. 
disette et l'accroissement de la pression fiscale consécutive à la guerre de Trente Ans. Les autorités de la ville s'efforcèrent au moins d'empêcher la progression du mal. Parlement et jurade constituèrent un Bureau de la santé chargé de coordonner les actions. Les finances de la ville furent mises à mal par les retours réguliers de ce fléau. Malgré un environnement difficile, de nombreuses mesures furent prises pour isoler la ville de l'extérieur, soigner les malades, désinfecter les maisons, nourrir les pauvres. L'efficacité des multiples règlements peut être mise en doute et c'est vraisemblablement d'elle-même que l'épidémie s'apaisa avant de revenir frapper au milieu du siècle, lors des troubles de la Fronde.

\section{La peste à Bordeaux}

Causes et déclenchement de l'épidémie

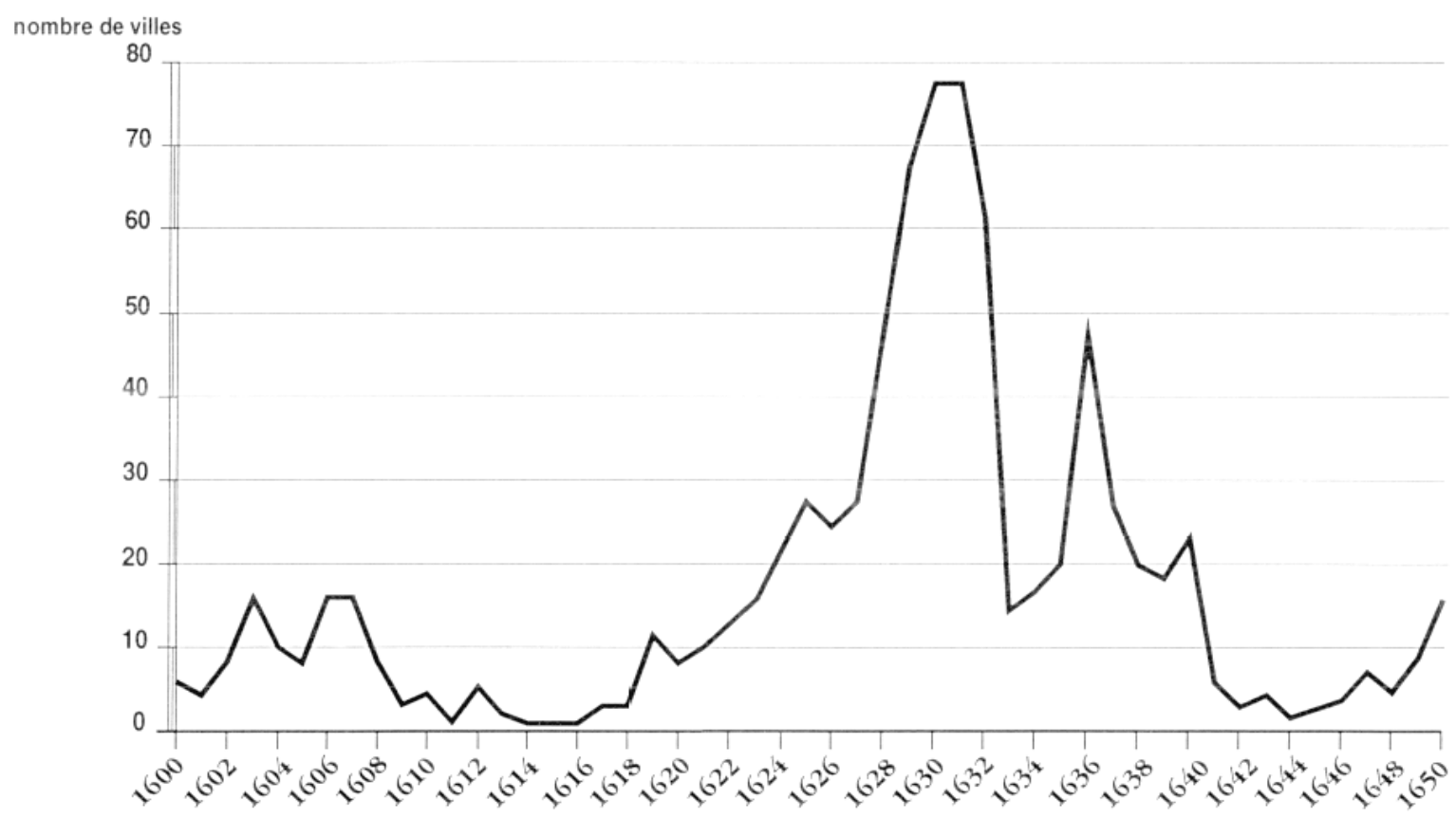

Fig. 1. - Villes françaises touchées par la peste, 1600-1650.

Lors des grandes poussées nationales de 1600-1616 et 1628-1632 (figure 1), Bordeaux ne fut pas épargnée mais les effets de la peste restent difficiles à cerner compte tenu d'une documentation très fragmentaire. Peu de registres paroissiaux antérieurs à mai 1606 ont été conservés et, si la période 1629-1631 est moins mal lotie, il n'est guère possible de reconstituer l'évolution démographique générale de la ville, faute de données dans l'ensemble des paroisses. Les autorités ayant fait enregistrer les maisons fermées pour cause de contagion, la progression géographique de la maladie, par contre, peut être bien suivie en 1604-1605. Les apparitions furtives de 1612, 1617, 1623, 1626 et 1628 


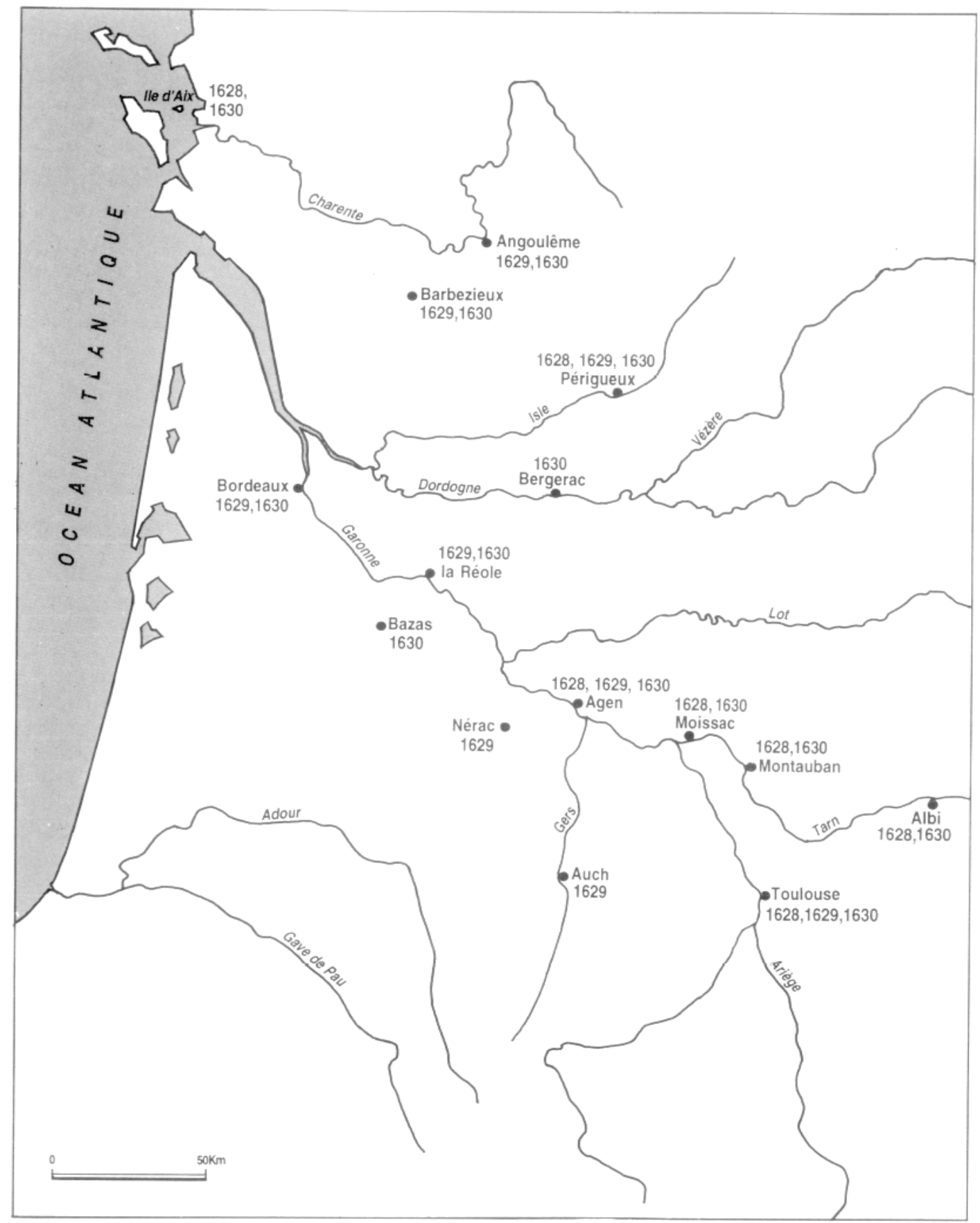

Fig. 2. - Villes du Sud-Ouest touchées par la peste de 1628 à 1630.

n'entraînèrent pas, autant que l'état de la documentation permet d'en juger, de mesures particulières. Pour 1629-1631, les registres remis le 14 janvier 1631 par l'intendant de la santé Vrignon n'ont pas été conservés ${ }^{5}$. Mais il ne subsiste aucune trace des activités du Bureau de la santé en 1635-1636 comme en $1645^{6}$.

5. Archives communales de Bordeaux (A.C.B.), GG 1214.

6. Ces institutions sont présentées dans la deuxième partie de cet article. 
Si l'origine de la peste semble à peu près sûre pour les contemporains en 1629 et 1636, il n'en est pas de même pour 1604. Pour la Chronique bourdeloi$s e$, la contagion commença au quartier Saint-Germain, au nord de la ville, où deux écoliers d'origine normande ${ }^{7}$ furent frappés avant que l'épidémie ne gagne du terrain. Pour Jean de Gaufreteau, au contraire, elle fut « portée de Tholoze avec des accoustrements e aultres hardes vendues ${ }^{8} \gg$. L'origine toulousaine fut également avancée en 1629 , la peste sévissant dans le haut pays depuis $1628^{9}$. La carte du Sud-Ouest (figure 2) montre bien la diffusion de l'épidémie à cette époque. À l'automne 1628, un arrêt du parlement de Bordcaux interdisait l'accès de son ressort aux Toulousains. À la même époque, les médecins bordelais conseillaient de tenir à l'écart les étrangers venant de lieux suspects. En février 1629, tout en confirmant le bon état sanitaire de la ville, ils conseillaient d'éviter la fréquentation des gens venant d'Agen et de Toulouse. Personne ne fait allusion à La Rochelle où la peste sévissait parmi les troupes royales alors qu'à plusieurs reprises, en 1627 et 1628 , la cité y avait envoyé des députés ou des messagers ${ }^{10}$.

Le 17 mai 1629, le Bureau de la santé, à peine constitué, mettait en cause les membres de la famille Castets, domiciliée près du fort du Ha, « pour avoir retirer dans leur maison des hardes infectées portées de la ville de Tholouse ${ }^{11}$ ». Trois ou quatre enfants du couple furent vraisemblablement les premières victimes de l'épidémie ${ }^{12}$. L'origine étrangère de la maladie prouvait ainsi l'inefficacité des mesures de quarantaine. La cause précise des vagues suivantes n'est pas évoquée par les contemporains. Lorsque, le 10 novembre 1635 , la contagion fut découverte dans le quartier Saint-Pierre, les médecins ne firent aucune mention de son lieu de provenance. Il en fut de même le 16 mars 1645, à une époque où un mal contagieux sévissait déjà aux alentours, dans le Blayais et le pays de Sainte-Foy.

\section{Le progrès du mal dans la cité}

Le mal rôdait et frappait mais en choisissant ses cibles. Le quartier SaintGermain, mis en cause par la Chronique bourdeloise en 1604, fut en réalité assez peu concerné, si l'on s'en tient aux informations fragmentaires du Bureau de la santé : une douzaine de maisons fut fermée entre la rue Porte-Dijeaux et

7. À Paris, au même moment, ce furent des étudiants qui furent accusés d'avoir mené l'infection d'Outre-Manche. J.-N. BIRABEN, op. cit., p. 92.

8. J. de Gaufreteau, Chronique bordelaise. Bordeaux, 1878, t. 2, p. 16.

9. A. Magen, « La ville d'Agen pendant l'épidémie de 1628 à 1631 d'après les registres consulaires ", Bulletin de la Société de médecine d'Agen, 1862.

10. A.C.B., BB 31.

11. A.C.B., GG 1214.

12. Un seul décès a été retrouvé dans les registres : « a esté ensevely [...] le corps de Françoise Castetz, agée de 10 ans, filhe demeurant ruhe des estubes ». A.C.B., GG 335. 
les remparts nord. Par contre le quartier méridional de Sainte-Croix, assez déshérité, fut touché dès le mois de mai 1604 : on y ferma neuf maisons durant le premier mois. Les paroisses du centre furent assez peu affectées ; seules la paroisse Sainte-Eulalie et les rues voisines du fort du Ha subirent ses assauts : vingt-neuf maisons y furent verrouillées par la jurade. À partir de janvier 1605 , (figure 3$)^{13}$, la maladie toucha toute la ville, mais la moitié sud resta la plus vulnérable : dix-huit maisons fermées rue Sainte-Croix, douze rue des Pommiers, sept rue du Ha. De mai 1604 à août 1605, 287 maisons au moins furent fermées, soit 19 par mois en moyenne. Pour celles dont l'adresse est connue, $45.4 \%$ sont localisées à Sainte-Croix et à Saint-Michel, et 29,8\% autour de Sainte-Eulalie.

La propagation de la peste était favorisée, outre le manque d'hygiène, par la promiscuité, l'entassement et la proximité de l'hôpital des pestiférés au sud de

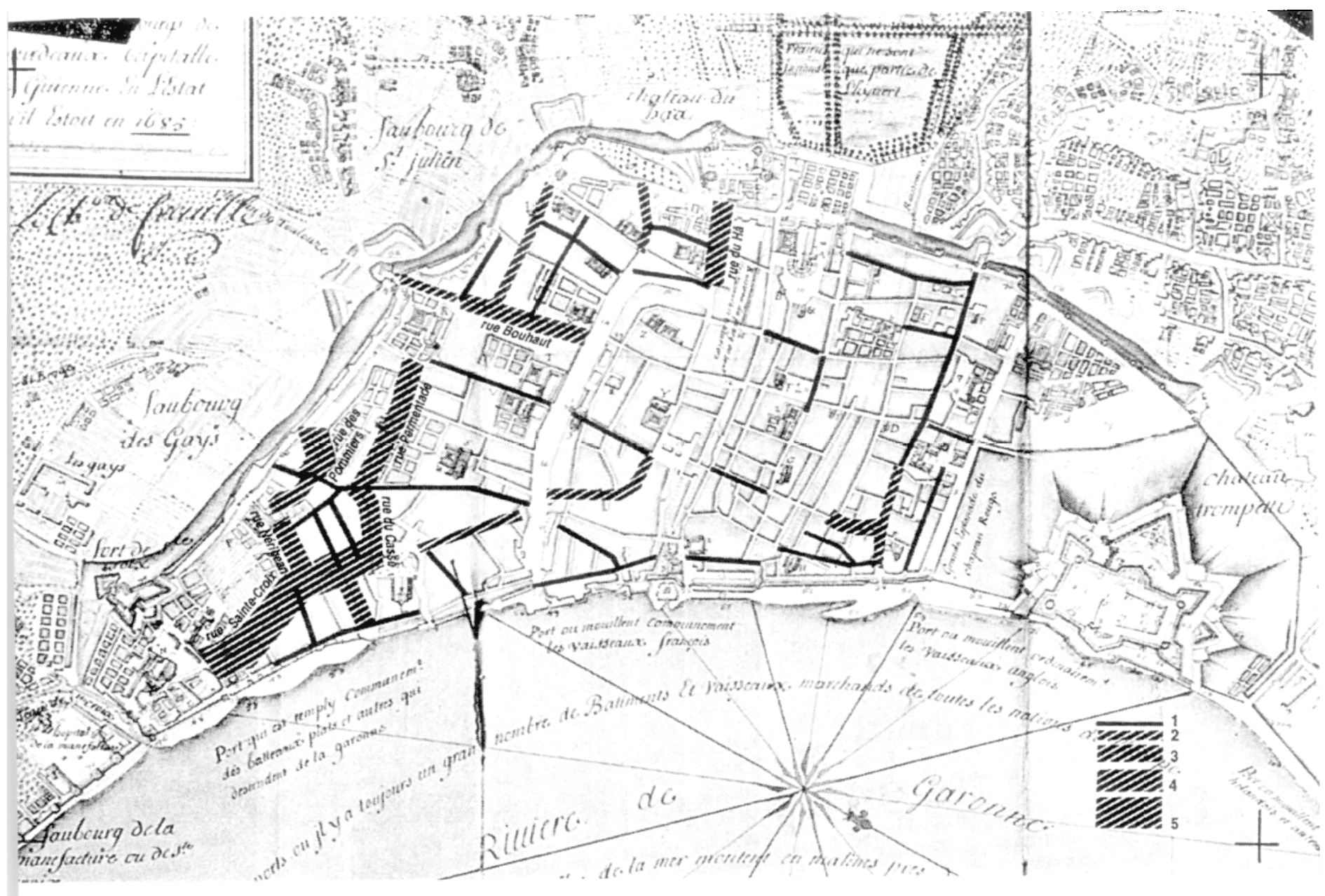

Fig. 3. - Nombre de maisons fermées par rues, de janvier à août 1605.

$1: 1 ; 2:$ de 2 à $5 ; 3:$ de 6 à $10 ; 5: 16$ et plus. 
la ville. Les consignes de quarantaine étant mal appliquées, des malades parvenaient plus ou moins clandestinement à entrer en ville, dans le quartier le plus proche, où les complicités de voisinage permettaient d'échapper à la surveillance des autorités. La peste sévissait donc à l'état endémique dans ces quartiers. La faiblesse de l'échantillon étudié ne permet guère d'être catégorique, d'autant que la fermeture des habitations ne signifie pas nécessairement le décès des occupants ; mais il semble que la propagation se soit intensifiée en novembre 1604 et de mars à mai 1605 , signe d'une possible peste pneumonique $^{14}$. Une évolution similaire se dessina en 1629-1631, mais la disparition des registres ne permet pas d'établir une chronologie de la fermeture des maisons. La géographie urbaine de la peste, moins précise, montre que les quartiers Sainte-Croix et Sainte-Eulalie furent, une fois de plus, les plus vulnérables ${ }^{15}$.

Toute peste n'est pas la peste car le terme pouvait désigner à l'époque toute maladie épidémique, ce qui rend aléatoire une étude des effets sur la population. Les délibérations font le plus souvent allusion au « mal contagieux » ou à la « contagion », mais quelques-unes, plus explicites, mettent en cause la peste. Depuis le milieu du XVIe siècle, le recours aux expertises médicales s'était peu à peu développé (à Lille en 1545, à Amiens en 1587, à Paris en 1604 par exemple) et de nombreux rapports de médecins et de chirurgiens bordelais font allusion en 1605, en 1629, en 1635 et en 1645 à la présence sur le corps des malades de bubons et de charbons, signes pathognomoniques les plus sûrs ${ }^{16}$. L'Histoire des Capucins rapporte que la mort du père Polycarpe de Marsiac au début du siècle avait été causée par " un charbon envenimé ${ }^{17}$ ». Ce fut aussi le cas pour une fille Castets examinée par le médecin Loppes dès le 14 mai 1629. Le rapport que le chirurgien Clavert présenta à la jurade le 18 juin suivant est le plus éloquent : il déclara avoir vu « une petite fille de neuf à dix ans quy avoict une enflure à la quisse et à cousté de l'oreille, comme aussy une autre fille de dix sept ou dix huit ans quy avoict quelque chosse de gros à cousté de l'œil droit ayantz des vomissemans et doleur de teste ${ }^{18}$ ». Dans la même maison un autre malade avait les " yeux eticelans ». Les symptômes présentés ici et dans d'autres rapports sont clairement ceux de la peste bubonique, caractérisée par la fièvre, des vomissements et des ganglions tuméfiés (bubons) au niveau de la piqûre de la puce ${ }^{19}$.

14. Celle-ci était transmise d'homme à homme par des gouttelettes de salive, les gouttelettes de Flügge.

15. Quelques noms de rues sont mentionnés dans les délibérations de 1636, sans que l'on puisse en tirer des conclusions probantes.

16. A.C.B., GG 1212, 1214 ; BB 32.

17. A.C.B., ms 583, Histoire des Capucins ou Memorabilia praecipua provinciae Aquitaniae sive tolosanae fratrum minoribus ordinis Sancti Francisci, Capucinorum, prae posteritati dicata, $f^{\circ} 22$.

18. A.C.B., BB 32.

19. V. FATTORINO, O. RitTER, Vademecum clinique du médecin praticien. Paris, 1985, p. 376377. 


\section{Des pertes humaines difficiles à chiffrer}

Les effets démographiques sont difficiles à quantifier pour Bordeaux alors que Lyon perdit la moitié de sa population en 1628-1629. Les témoignages parlent certes de nombreuses victimes mais insistent surtout sur la durée de la contagion. Si, selon dom Devienne, l'on tint un registre des morts de la peste en 1585, il n'en fut pas de même ou l'on n'en garda pas la trace au $\mathrm{XVII}$ siècle. Compte tenu du nombre de maisons fermées, on peut estimer qu'il y eut quelques centaines de victimes directes ${ }^{20}$ au maximum en 1604 -

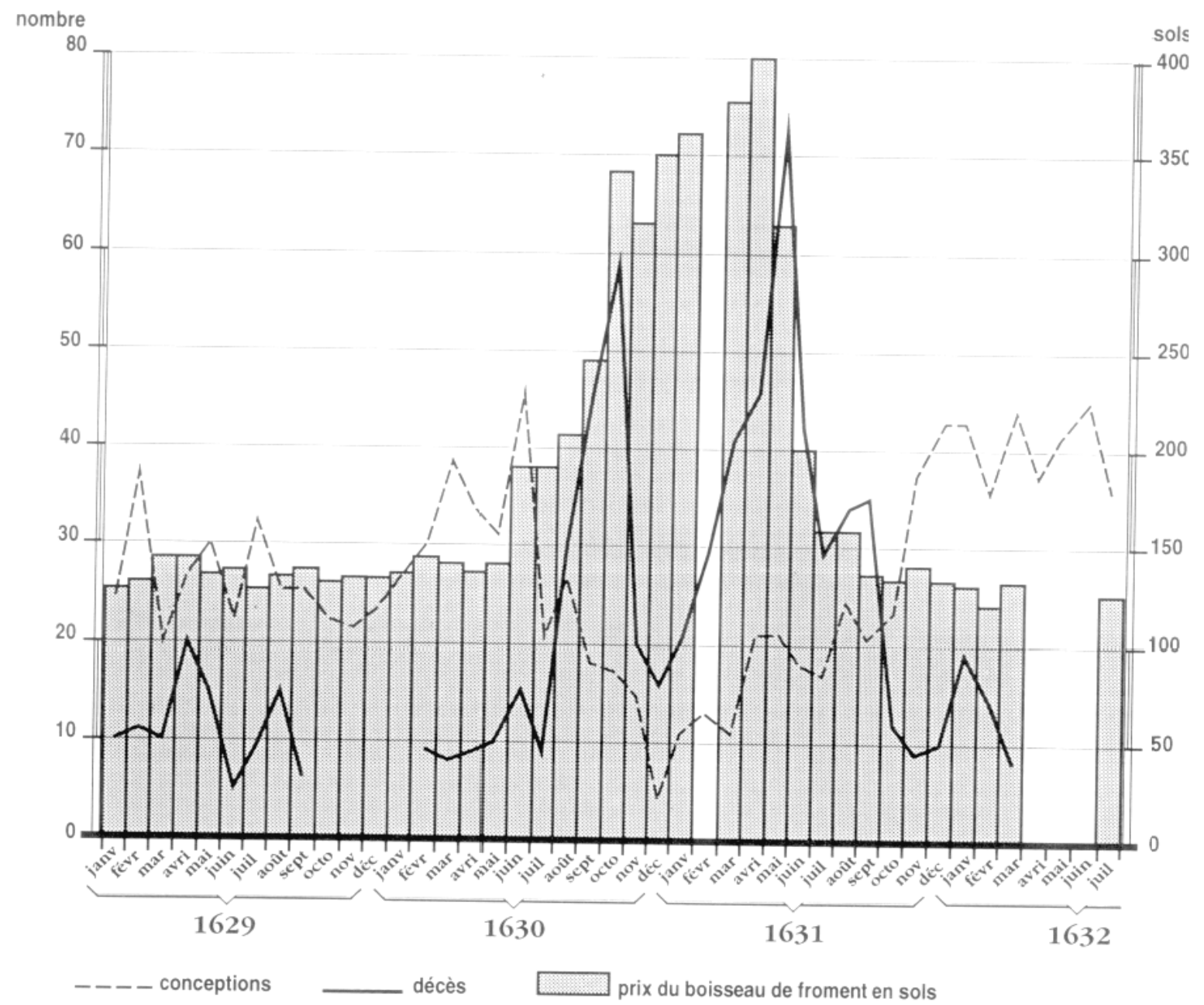

Fig. 4. - Évolution des conceptions, des décès et du prix du boisseau de froment à Sainte-Croix, 1629-1632.

20. Tous les décès n'étaient pas notés correctement à l'époque et la peste venait interrompre l'enregistrement par les curés, à Bordeaux comme ailleurs (Lyon en 1628, Riom en 1631 ; J.-N. BiRABEN, op. cit., p. 113). Nombreux étaient les cadavres enterrés à l'extérieur de la cité, de préférence la nuit, par les croquemorts de la ville : « corbeaux » à Lyon, « mouthonniers » à Nevers, ils étaient appelés « crocs " à Bordeaux ; ceux mentionnés dans un acte notarié de 1612 étaient laboureurs. Archives départementales de la Gironde (A.D.G.), 3 E 3943, fo 362. 
1605. En 1629-1630, les rapports - incomplets - du Bureau de la santé et les indications de certains registres paroissiaux donnent un minimum de 233 décès dus à la peste. Quant à la diminution de la population, elle était surtout consécutive au fait que « la plus grand partye du peuble estoit aux champz ${ }^{21}$ », c'està-dire à la fuite de certains habitants.

La contagion ne peut toutefois être tenue pour seule responsable de la crise, car les témoignages insistent sur la conjonction de l'épidémie et de la disette. La célèbre formule « a fame, peste, bello, libera nos, Domine » peut s'appliquer mais, contrairement à ce qu'écrit le biographe du gouverneur de la Guyenne ${ }^{22}$, c'est la disette qui succéda à l'épidémie et causa le plus de ravages. La mise en corrélation des courbes des prix des céréales fournis par le visiteur des grains avec celle des conceptions et des décès est particulièrement instructive (figure 4$)^{23}$. Au début de l'épidémie, de mai 1629 à mai 1630, le prix du boisseau de froment oscille autour de sept livres. On constate bien une chute des conceptions à Sainte-Croix mais elle n'a rien de particulièrement marqué, et en hiver, au moment où la peste régresse -59 décès dans les hôpitaux en septembre 1629,18 en décembre -, on observe une légère reprise. Au printemps 1630, par contre, deux crises se juxtaposent. L'épidémie reprend, vraisemblablement avec moins de virulence, et, à la suite de mauvaises récoltes dues à la sécheresse et à la désorganisation de l'économie, les prix du froment s'envolent, atteignant 22 livres le boisseau en avril 1631. À ce moment-là les conceptions ont déjà commencé leur redressement, qui s'accentue avec la baisse des prix. À Sainte-Eulalie, où la mortalité paraît très mal enregistrée en 1629, la montée des prix entre mai 1630 et avril 1631 s'accompagne d'une forte mortalité, entrecoupée d'un répit au cours de l'hiver. La faible alimentation eut sans doute raison des gens convalescents ou malades, sans omettre les méfaits de la mauvaise alimentation notée par les chroniqueurs. "Infinité de peuple mouroit par les rues et mangeoit des herbes mortifères, mesme les charognes aussi ${ }^{24} »$, écrit Jean de Gaufreteau. Des rumeurs d'anthropophagie atteignirent même La Réole : «Eodem anno », notent les moines, « annonae tam grandis penuria fuerit per totam Aquitaniam, ut saccus frumenti viginti aureis venderetur ; urgenteque rabida fame, inventi sunt, qui cadaveribus equorum, canum, felium, et [...] humana carne vescerentur ${ }^{25}$. » Malgré l'arrivée de grains de Bretagne, la pénurie de farine et de pain restait grande, ce qui accentuait la « plainte du peuple ${ }^{26}$ ». À l'opposé, la raréfaction des allusions à la maladie tendrait à montrer une accalmie de l'épidémie en 1631. Le 3 janvier 1632, les

21. A.C.B., BB 32.

22. GIRARD, Histoire de la vie du duc d'Espernon, Paris, 1655, p. 459-460.

23. Notre échantillon est certes limité à deux paroisses (Sainte-Croix et Saint-Michel) mais il concerne des quartiers particulièrement touchés si l'on s'en tient au registre des maisons fermées.

24. J. de Gaufreteau, op. cit., p. 171.

25. Sancti Petri de Regulo regalis prioratus historico-rhronologica sinopsis, publié dans Archives historiques de la Gironde. Bordeaux, 1901, t. 36, doc. $\mathbf{n}^{\circ} 1$, p. 71.

26. A.C.B., BB 32, 33. 
jurats annoncaient officiellement que la maladie avait cessée ${ }^{7}$, même s'il y eut quelques alertes en 1633 et 1634 . La prudence s'imposait et d'ailleurs, à la suite du décès du maréchal Henri de Schomberg à l'automne 1632, la reine et Richelieu, «craignant qu'il n'y eut de la maladie contagieuse ${ }^{28}$ ", abrégèrent leur séjour dans une ville qu'ils crurent à nouveau menacée. Le 10 novembre 1635, la contagion fut constatée à Saint-Pierre ${ }^{29}$, au moment où la cherté se développait, mais à un niveau très nettement inférieur à celui de 1630-1631. Les cas furent vraisemblablement moins nombreux et dès l'hiver 1636-1637 la maladie s'effaça à nouveau. En 1644-1646, la Chronique bourdeloise reconnaît qu'il « y eut quelques gens qui moururent de la maladie contagieuse ${ }^{30}$ » mais ne s'y attarde pas, car la disette avait causé des ravages bien plus graves. La cherté avait en effet précédé l'épidémie, puisque le boisseau de froment s'échangeait à cinq livres le 10 novembre 1645 , lorsque la contagion fut découverte dans le quartier Saint-Pierre, alors que le maximum avait été atteint bien avant, entre octobre 1643 et janvier 1644, avec 13 livres. Ainsi, chaque vague de maladie frappait dans un contexte différent. Avec la Fronde, la peste vint menacer à nouveau la ville, dans des circonstances qui mériteraient des études plus approfondies.

\section{Les autorités face la peste}

\section{Parlement, gouverneur et jurade}

Les autorités ne pouvaient rester inertes face au mal et à ses conséquences. Mais une coopération s'avérait nécessaire dans une grande ville où plusieurs juridictions concurrentes pouvaient se neutraliser en cas de conflit de compétence.

Le parlement de Bordeaux fut un des éléments-clés de la lutte contre le fléau. Ayant en charge l'ordre public, il pouvait, de sa propre initiative, ou à la demande des autres autorités locales, prendre des arrêts pour lutter contre la maladie, mais il devait nécessairement élaborer ses décisions en accord avec ceux qui étaient chargés de les exécuter. Seuls quelques parlementaires participaient à l'effort commun, car en 1604 comme en 1629 ou en 1636 l'activité normale des cours s'interrompit à cause de l'épidémie.

Le gouverneur, quant à lui, était régulièrement informé par le corps de ville, qu'il résidât à Bordeaux, ou, pour le duc d'Epernon, dans son château de Cadillac qui reçut la visite de plusieurs délégations de jurats. On recherchait ses conseils, qui furent peu originaux (discrétion, expulsion des mendiants, net- 
toyage des rues), mais surtout les appuis dont il disposait à la cour ou auprès des gouverneurs d'autres provinces épargnées par la disette et l'épidémie. Le rôle de Jean-Louis de Nogaret de La Valette, duc d'Épernon, sans être négligeable, fut moins déterminant toutefois que ne le laisse entendre son biographe. Informé régulièrement par la jurade, il mit tout en œuvre «pour réprimer la licence des soldats dans le plat païs » et usa de son crédit pour « faire venir les secours des bleds ${ }^{31} \gg$.

Les autorités municipales avaient la responsabilité de l'approvisionnement, de la salubrité et de la sécurité de la cité. Le pouvoir était détenu par les six jurats, par le procureur syndic et par le clerc de ville, et par le maire en 16041605. Le maréchal Alfonso d'Ornano, maire de 1599 à 1610 , a joué un rôle important dans la lutte contre la peste, donnant de sa personne, mettant à la disposition de la ville les soldats du Château Trompette, offrant 2000 à 3000 écus pour subvenir aux besoins ${ }^{32}$. Mais il apparaît peu dans les registres de délibérations de la jurade et du Bureau de la santé. La place de maire étant vacante par décision de Louis XIII depuis septembre 1620, les crises ultérieures incombèrent aux seuls jurats. Ceux-ci, deux gentilshommes, deux avocats et deux bourgeois, en place pour deux ans, étaient renouvelés par moitié au début de chaque mois d'août. S'il n'est pas possible de retracer l'attitude de la mairie au début du siècle, les registres de délibérations ayant été perdus d'août 1604 à janvier 1610 , il n'en est pas de même pour la deuxième grande épidémie qui mobilisa les énergies : 479 réunions au moins furent tenues entre mai 1629 et août 1632 , soit une douzaine par mois. Ce rythme épuisa une partie des édiles : si le procureur syndic et le clerc de ville assistèrent à la quasi-totalité des séances, on ne compta en moyenne que quatre jurats, les bourgeois étant nettement plus assidus que les gentilshommes, qui n'assistèrent qu'à la moitié des réunions. Sur les quinze jurats qui eurent successivement en charge les affaires de la cité pendant la crise, six seulement vinrent à plus des quatre cinquièmes des séances, ce qui est mieux qu'à Lyon, où un seul échevin resta en ville en $1628^{33}$.

Dans des circonstances graves comme celles-ci, les jurats usaient de leur pouvoir de réunir les Cent et Trente, organe délibératif qui regroupait le Conseil des Trente, choisi tous les ans par les jurats lors de leur installation, et une centaine de notables, bourgeois, avocats, anciens juges, consuls de la Bourse et citoyens, c'est-à-dire anciens jurats. La réunion était à la discrétion des jurats, qui refusèrent ainsi le 28 février 1630 que l'on débatte d'une députation dont la composition déplaisait aux milieux du négoce ${ }^{34}$. Mais, à l'opposé,

31. GiRARD, op. cit., p. 460.

32. Chronique bordelaise, p. 125. L. FILIPPI, Essai sur le maréchal de France Alfonso d'Ornano, maire de Bordeaux (1548-1610), Alger, 1915, p. 90-91.

33. J.-N. BIRABEN, op . cit., p. 164.

34. Un conflit opposait depuis le début de l'année la jurade et les juges et consuls de la Bourse pour la désignation d'un député de robe courte pour étoffer une délégation envoyée vers le roi en novembre 1629. A.C.B., BB 32. 
le 13 décembre suivant, une séance dut être reportée faute de quorum. La plupart des sources évoquent l'absence des principaux bourgeois, qui avaient gagné leur maison de campagne pour rejoindre les membres de leur famille mis à l'abri de l'épidémie.

Lors de chaque alerte, les jurats s'empressaient d'envoyer une délégation au parlement. Le cas échéant, ce dernier s'informait auprès de la municipalité. La maîtrise de la police de la ville suscita bien quelques réactions de défiance entre jurats et magistrats. Jalouse de son indépendance, la jurade réaffirma ses prérogatives en 1604 et surtout en août 1629, lorsqu'une assemblée des trois ordres se réunit à l'initiative du président aux enquêtes Gilles de Favard. Ces incidents mis à part, la coopération entre le parlement et la jurade s'opéra sans trop de difficultés au sein d'un Bureau de la santé. Celui-ci, attesté à Bordeaux en 1604, avait déjà été mis en place auparavant dans d'autres villes du royaume, comme Lyon en 1564, Nîmes en 1586 ou Amiens en 1596.

\section{Le Bureau de la santé}

Chargé de connaître l'état sanitaire de la ville, d'assurer la police, de surveiller les dépenses de santé, et constitué par le parlement à la demande des magistrats municipaux, le Bureau se réunissait une à deux fois par semaine à l'hôtel de ville ou dans la demeure d'un magistrat, comme en 1629 chez le président Daffis lorsque les prisons de la mairie furent contaminées. Sa mise en place fut plus ou moins rapide : si l'on en croit Charles de Cadouin ${ }^{35}$, jurat en 1605 , la peste apparut en ville dès le 5 octobre 1603 mais le Bureau ne fut constitué que le 16 décembre 1604 . De même, la peste fut révélée le 10 novembre 1635 et ce n'est que le 19 juillet 1636 que la jurade sollicita la création d'un Bureau dont les archives ont été perdues ${ }^{36}$. En 1645, du fait d'une moindre menace, le Bureau ne joua pas de rôle décisif car un rapport de la jurade indique qu'il ne s'était pas réuni de novembre 1645 à mars 1646, alors que les autorités avaient été alertées par les médecins dès le 10 novembre $1645^{37}$. Par contre, en 1629, la réaction fut plus rapide, car la première réunion eut lieu dès le 17 mai, peu de temps après la découverte des premiers cas.

$\mathrm{Si}$, en 1604-1605, les témoignages s'accordent pour donner trois représentants à la magistrature - le président Gabriel de Tarneau, les conseillers Geoffroy de Montaigne et Jacques de Lescure -, ils divergent sur le nombre des jurats : trois pour la Chronique bourdeloise, quatre selon les registres plus fiables du Bureau de la santé. En 1629, celui-ci comprenait douze membres: les huit représentants du corps de ville et quatre magistrats, un président, deux conseillers et l'avocat ou le procureur général du roi. De 1629 à 1632, le 
Bureau se réunit au moins 85 fois $^{38}$, mais il ne fut complet, à notre connaissance, qu'une seule fois. On comptait en moyenne neuf participants, à peu près autant de magistrats que de jurats, mais le procureur syndic et le clerc de ville faisaient pencher la balance en faveur de la mairie. Les jurats semblaient donc contrôler les débats, puisqu'ils ne changeaient que tous les deux ans et se réunissaient par ailleurs à l'hôtel de ville très fréquemment. Les vingt-quatre conseillers et les sept présidents qui vinrent à tour de rôle n'eurent pas le même suivi. Seuls six conseillers assistèrent à plus de dix réunions. Les présidents Jean Daffis, François de Pichon, le procureur Jean de Pontac et l'avocat général Du Sault vinrent suffisamment souvent pour maîtriser les dossicrs que les jurats présentaient par ailleurs à la cour. Le Bureau ne se réunissait pas seulement en petit comité ( 39 fois), il s'ouvrait aussi ( 42 fois) à des personnalités intéressées au premier chef par la lutte contre la peste ${ }^{39}$, médecins comme François Loppes et Jean de Maures, professeur à la faculté, chirurgiens, intendants de la santé, bourgeois, en moyenne quatre à cinq personnes mais dix-huit le 5 juillet 1629. Ainsi, onze à douze personnes en moyenne se réunissaient à la mairie pour suivre l'évolution du mal et prendre les mesures dictées par la situation (figure 5).

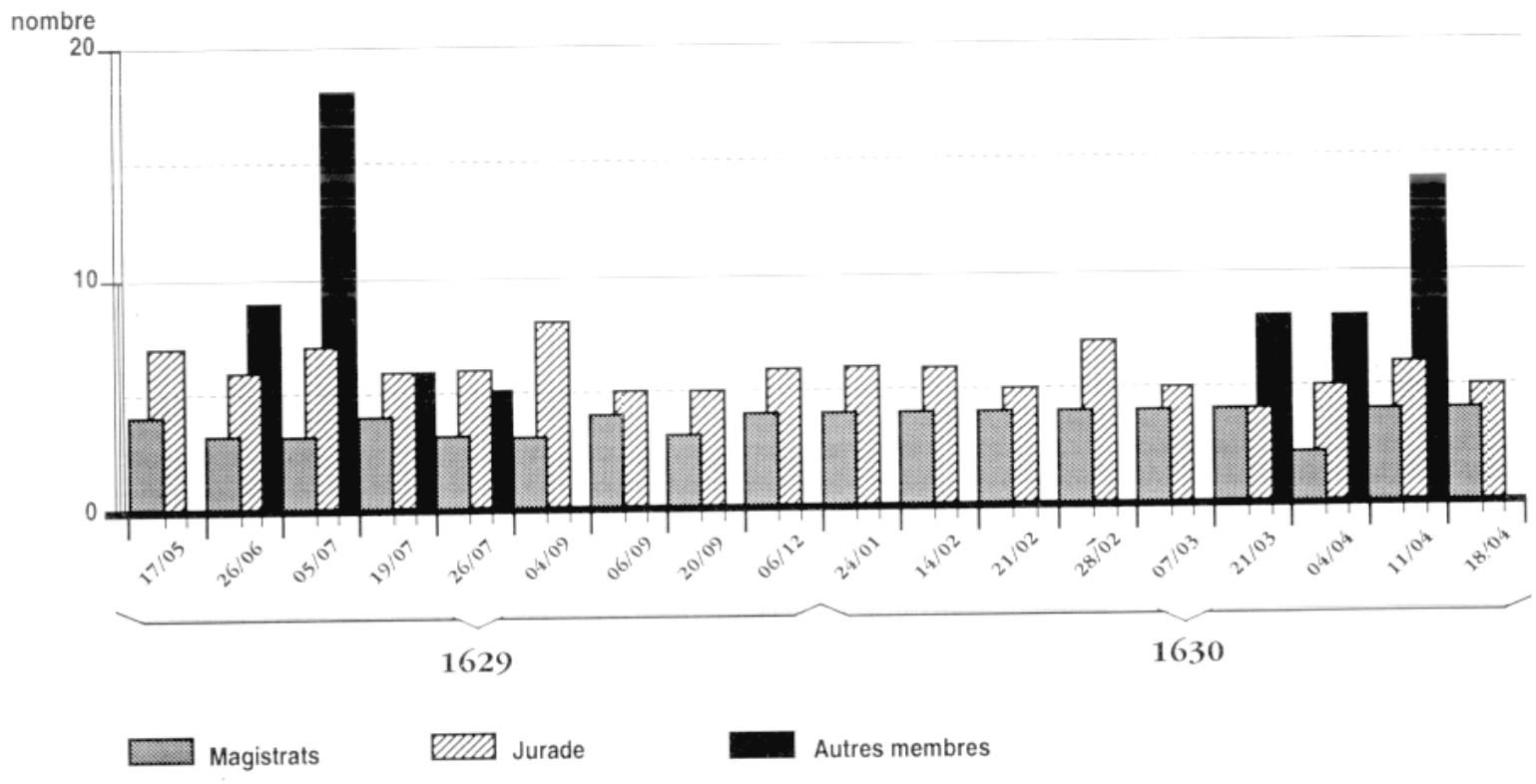

Fig. 5. - Réunion du Bureau de la santé, mai 1629-avril 1630.

Les décisions prises par le parlement, la jurade ou le Bureau de santé nécessitaient un personnel d'exécution qui, comme nous l'avons vu, participa épisodiquement à l'élaboration des règlements. Du fait d'une titulature assez fluc-

38. Neuf fois de mai à août 1629,42 fois d'août 1629 à août 1630,24 fois d'auût 1630 à août 1631,7 fois d'août 1631 à août 1632 et 3 fois à des dates inconnues.

39. On ne connaît les membres présents que pour 81 réunions. 
tuante, on ne peut brosser qu'un tableau sommaire de l'administration subalterne. Le personnage-clé fut Mathurin Vrignon. Ancien consul et juge de la Bourse, jurat de 1620 à 1622 et à ce titre citoyen de Bordeaux, il exerça les responsabilités de commissaire et intendant de la santé ${ }^{40}$ du début de l'épidémie à l'été 1631 , où il fut remplacé, à sa demande, par Pierre Deleys. Il assista à trente-deux réunions du Bureau, présentant l'état de la santé, rédigeant les registres de maisons fermées, supervisant les dépenses dont il faisait souvent l'avance. Il fit le lien entre les autorités supérieures et les simples exécutants. Pour les autres agents, la titulature varie d'un jour à l'autre pour le même individu, ce qui ne permet guère de décrire les fonctions. On comptait un nombre variable de capitaines de la santé, une quinzaine de commissaires de la santé appelés aussi commissaires de police, dont certains avaient vraisemblablement exercé les charges de juge ou de consul de la Bourse. Les hôpitaux chargés d'accueillir et de soigner les pestiférés étaient sous la responsabilté des intendants des hôpitaux Pierre Deleys et Martial Duchambon. En 1629, on décida d'élire dans chaque jurade ${ }^{41}$ des dizeniers chargés, en quelque sorte, de quadriller le terrain, d'informer les jurats sur la présence d'individus suspects et sur le non-respect des arrêts. Le 16 novembre 1629, 97 dizeniers au moins prêtèrent serment. La profession, connue pour 32 d'entre eux, montre un recrutement chez les petits notables de la ville puisqu'on dénombre dix notaires, six procureurs, quatre sergents royaux, trois bourgeois, deux apothicaires ${ }^{42}$. Mais le recrutement variait selon les quartiers et l'efficacité s'en ressentait. Le 20 décembre 1629, le Bureau de la santé chargeait d'Aiguille, jurat de SaintMichel, et Cazenave, jurat de Sainte-Croix, quartiers les plus touchés, de choisir des bourgeois pour surveiller les dizeniers qui, « estans des pauvres gens et de peu de considération ${ }^{43}$ », étaient peu obéis dans ces deux paroisses. De fait, la fonction de dizenier, conçue comme une surveillance rapprochée, ne devait être guère recherchée et il fallait régulièrement en changer. Aussi procéda-t-on, en octobre-novembre 1630 et en juin 1631 , à de nouvelles nominations ${ }^{44}$. Les autorités pouvaient compter également sur le guet et sur les douze sergents ordinaires de l'hôtel de ville. Les jurats, et en 1605 le maire Alfonso d'Ornano, participèrent à la mise en œuvre des décisions, en inspectant, à tour de rôle, seuls ou escortés de magistrats et de bourgeois, leur jurade et les hôpitaux.

40. Il s'agit vraisemblablement de celui que les statuts de Bordeaux appellent le « capitaine de la peste ", un " homme de bien ". Anciens et nouveaux statuts de la ville et cité de Bordeaux. Bordeaux, Boé, 1701, p. 128.

41. La ville était partagée en six quartiers appelés jurades puisque chacun des membres du corps municipal en avait la responsabilté. Cet usage, encore vivace au XVII siècle, semble être devenu honorifique à la fin de l'Ancien Régime.

42. Mais aussi deux greffiers, un huissier, un boulanger, un boucher, un marchand et un orfèvre. 43. A.C.B., GG 1214 .

44. A.C.B., GG 1211. Parmi lesquels trois notaires, deux marchands et deux procureurs. 


\section{Les aspects financiers et économiques}

Compte tenu du poids financier de la lutte contre l'épidémie, Dordé Taurisson, trésorier ordinaire de la cité, fut secondé, en 1629-1632, par deux trésoriers des deniers de la santé. Ce furent Arnaud Dumalle, ancien juge de la Bourse, et François Fouques. Dumalle fut remplacé en novembre 1630 par Géraud Olivier, après son élection comme jurat bourgeois. Dès le 3 août 1630, il avait d'ailleurs associé son collègue Fouques à sa promotion, en le nommant membre du Conseil des Trente ${ }^{45}$. La charge de trésorier de la santé n'était pas de tout repos et les candidats n'étaient pas légion. Si Jean de La Mothe accepta de l'être en septembre 1636, ce fut sous la pression des jurats ${ }^{46}$. En 1645-1646, ce fut même à un jurat qu'incomba cette charge, malgré les réticences de son corps qui préférait un bourgeois à ce poste ${ }^{47}$.

En collaboration avec Vrignon en 1629-1631 et sous la surveillance étroite des jurats et du parlement, ils durent gérer le financement des opérations. À un échelon inférieur, opéraient deux receveurs et deux distributeurs des deniers des pauvres nécessiteux, douze collecteurs et douze distributeurs des pauvres, nommés par les jurats ${ }^{48}$.

La peste représentait en effet un défi majeur pour l'économie et les finances d'une ville. Les recettes diminuaient, les dépenses s'accroissaient dans des proportions considérables et le recours obligé à l'emprunt entraînait un endettement pour de longues années.

Les revenus de la ville s'effondraient du fait de la mortalité, de la fuite des habitants et de la diminution de l'activité économique. Les marchands et négociants devaient accepter l'interruption plus ou moins longue de leurs relations commerciales avec les villes suspectes, comme Toulouse et Lyon en 1629. On conçoit que les autres villes de la province et du royaume cessaient également leurs relations avec Bordeaux. Les ruraux présents pour leurs procès étaient renvoyés car, à chaque crise, le 17 août 1605, le 3 août 1629, en été 1636, le parlement suspendait les audiences et congédiait les parties ${ }^{49}$. Le 16 novembre 1630 , le bureau des finances de Guyenne décidait d'ajourner ses réunions à des jours meilleurs $^{50}$. Les collèges étaient fermés. Cette perte de revenus pour les marchands, les avocats, les hôteliers, se répercutait sur la ville. Les fermiers des domaines et droits de la ville s'empressaient de réclamer des rabais pour cause de contagion, puis, en 1631 , de disette. Dès septembre 1629, la jurade accordait un rabais de $8,25 \%$ à Pierre Thomas, le fermier du droit des échats ${ }^{51}$. Cinq rabais au moins étaient accordés en 1629, quatre en 1630, six en 1631 et

45. A.C.B., BB 33.

46. A.C.B., BB 36.

47. A.C.B., BB 43.

48. A.C.B., BB 32.

49. A.C.B., BB 32 ; Chronique d'Érienne de Cruseau, Bordeaux, 1881, t. 2, p. 2.

50. A.D.G., C $3907, \mathrm{f}^{\circ} 152$.

51. Droit de six pots par barrique perçu sur le vin vendu en taverne. 
quatre encore en février 1632 alors que l'épidémie et la disette s'éloignaient Les jurats accordaient en règle générale un rabais d'un quartier $(25 \%)$ et les requêtes étaient rarement repoussées. Le montant des rabais est très raremen précisé, ce qui ne permet pas, compte tenu de l'état des registres comptables. d'estimer les pertes totales de la caisse municipale. Les rabais ne sont pas seuls en cause. Les enchérisseurs ne se bousculaient pas pour prendre à ferme les revenus urbains : un seul se présenta en janvier 1630 pour les terres limitrophes de l'hôpital de l'Enquesteur et la jurade fut contrainte de signer avec lui. Il fallut même, le 17 novembre 1631, consentir à adjuger une partie des domaines pour trois ans au lieu d'un afin d'attirer des candidats.

À l'opposé, les dépenses connaissaient un net accroissement : il fallait recruter davantage de gardes, acheter des médicaments aux apothicaires, rétribuer les médecins, le personnel et les intendants des hôpitaux. Mathurin Vrignon devait avancer les frais, dont il était, plus ou moins régulièrement, remboursé par le trésorier des deniers de la peste, dont la caisse était alimentée par la caisse de la ville, elle-même en piteux état. En 1631, il fallut acheter des quantités importantes de blé pour éviter la famine et les troubles inhérents à la misère. La peur des émotions populaires était justifiée. En mai 1631, les jurats, informés d'un projet de soulèvement fomenté par « certains habitans malaffectionnés $^{52} »$, firent renforcer les patrouilles du guet. Quelques années plus tard, en août 1643, lors des chertés, le jurat Minvielle, que la foule voulait égorger, se réfugia au fort du Hâ et ne fut sauvé que par la distribution de pain aux émeutiers $^{53}$. La disparition ou l'état très fragmentaire des registres de comptabilité, particulièrement endommagés, ne permettent pas de mesurer le surcroît de frais mais il ne put être que conséquent. La conjoncture militaire n'était guère plus favorable avec le siège de La Rochelle, les combats en Italie et la guerre de Trente Ans. Les villes devaient contribuer à l'effort de guerre : en 1627, la jurade avait déjà participé à l'équipement de la marine ; en juin 1629 il fallut acquitter les frais de nourriture de 2000 soldats qui allaient de Royan à Preignac $^{54}$; en 1630 , la ville fut taxée de 11000 livres au profit des garnisons de Casal et de Suse ${ }^{55}$.

Il fallut donc, dans ces circonstances pénibles, trouver des recettes exceptionnelles. En 1629-1631, le parlement créa deux maîtrises dans chaque corps de métier pour alimenter la caisse des pestiférés. Les contraventions à la taxe du pain, aux règlements d'hygiène, à la fermeture des maisons, permettaient d'encaisser des amendes ${ }^{56}$, mais cela ne suffisait pas et l'on devait taxer régulièrement la population pour aider les pestiférés. Les jurats nommaient des bourgeois pour dresser des rôles de contribuables et collecter les fonds ${ }^{57}$, tâche

52. A.C.B., BB 33.

53. Inventaire sommaire des registres de la jurade, Bordeaux, 1901, vol. 2, p. 343-345.

54. A.C.B., BB 32.

55. Y.-M. BERCÉ, «La bourgeoisie bordelaise et le fisc sous Louis XIII », Revue historique de Bordeaux, 1964, p. 45.

56. A.D.G., 12 B 4, 12 B 6.

57. $C f$. note 40 . 
J'autant plus ardue que nombreux étaient ceux qui avaient fui à la campagne, à l'abri de la peste et des obligations qui pouvaient leur incomber. Les différents zorps de la ville furent alors mis à contribution. Ainsi, le 31 décembre 1630 , les trésoriers généraux de France, les receveurs et les contrôleurs généraux s'engagèrent à entretenir 140 pauvres désignés par les jurats ${ }^{58}$. En mars 1646 , une quête fut organisée au sein des compagnies et des métiers. Le recours à l'emprunt s'imposa dès les débuts de la grande épidémie. De juin 1629 à janvier 1630 , la ville sollicita d'emprunter au moins 45300 livres tournois ${ }^{59}$. Les registres du trésorier, fort endommagés en 1862, gardent cependant la trace de contrats passés devant notaire entre la ville et un certain nombre de riches notables, le plus souvent des parlementaires, qui purent tirer d'affaire le corps de ville, en faisant de bons placements ${ }^{60}$.

Ces quatorze contrats répertoriés (figure 6), car il y en eut sans doute d'autres, représentent un capital de 73100 livres prêtées au denier quinze.

Malgré ces soutiens importants, la situation financière restait fragile. Un compte présenté par le trésorier le 24 novembre 1605, pour une demi-année, donne 2929 livres de déficit, avec un taux de couverture de 93,3\%; un autre, non daté mais vérifié sans doute en septembre 1630 , présente un déficit de 5505 livres, soit un taux de couverture de 85,5\% mais pour une période inconnue. La peste, par son ampleur et par son retour fréquent, obérait donc les finances locales pour de longues périodes. En 1645, par exemple, les intérêts

58. A.D.G., C 3907, $\mathrm{f}^{\circ}$ 170. À Lyon, à la même époque, les portes des riches portaient des placards avec le nom des pauvres qu'ils devaient entretenir.

59. En juin 162911000 l. au chapitre Saint-André ; en septembre 70001 . : en novembre 12300 l. ; en janvier 1630150001 .

60. A.C.B., CC 27, 33. Archives historiques de la Gironde. 1. 19, 1879, n 214, p. 551-552.

\begin{tabular}{|c|c|c|c|}
\hline Date & Nom du créancier & Fonctions & Montant du prêt \\
\hline $10 / 08 / 29$ & F. de Métivier & $\mathrm{C}^{\mathrm{r}}$ au Plt & 30001 \\
\hline $10 / 08 / 29$ & J. -G. de Voisin & $\mathrm{C}^{\mathrm{r}}$ au $\mathrm{Plt}^{\mathrm{t}}$ & 90001. \\
\hline $8 / 09 / 29$ & D. Dubourg & $C^{r}$ au plt & 16001 \\
\hline $20 / 09 / 29$ & M. Bonneau, veuve & Libraire & 30001 \\
\hline $28 / 09 / 29$ & Veuve de Laserre & $\mathrm{C}^{\mathrm{r}}$ au Plt & 70001 \\
\hline $9 / 11 / 29$ & P. de Mullet & $\mathrm{C}^{\mathrm{r}}$ au $\mathrm{P}^{\mathrm{lt}}$ & 70001 \\
\hline $26 / 11 / 29$ & Faure & Bourgeois & 45001 \\
\hline $12 / 12 / 29$ & Syndic de l'hôpital Saint-André & & 20001 \\
\hline $31 / 12 / 29$ & P. de Mullet & $\mathrm{C}^{\mathrm{r}}$ au $\mathrm{P}^{\mathrm{lt}}$ & 10001 \\
\hline $22 / 01 / 30$ & J. Desbaras & $S^{e}$ du Roi & 60001 \\
\hline $30 / 03 / 30$ & Veuve de Brezets & $\mathrm{C}^{\mathrm{r}}$ au Plt & 50001 \\
\hline $5 / 12 / 30$ & M. de Francs & $\mathrm{C}^{\mathrm{r}}$ au Plt & 180001 \\
\hline $5 / 03 / 31$ & M. Cazenave & & 40001 \\
\hline $4 / 04 / 31$ & J. Daffis & $\mathrm{P}^{d t}$ au $\mathrm{P}^{\mathrm{lt}}$ & 10001 \\
\hline
\end{tabular}




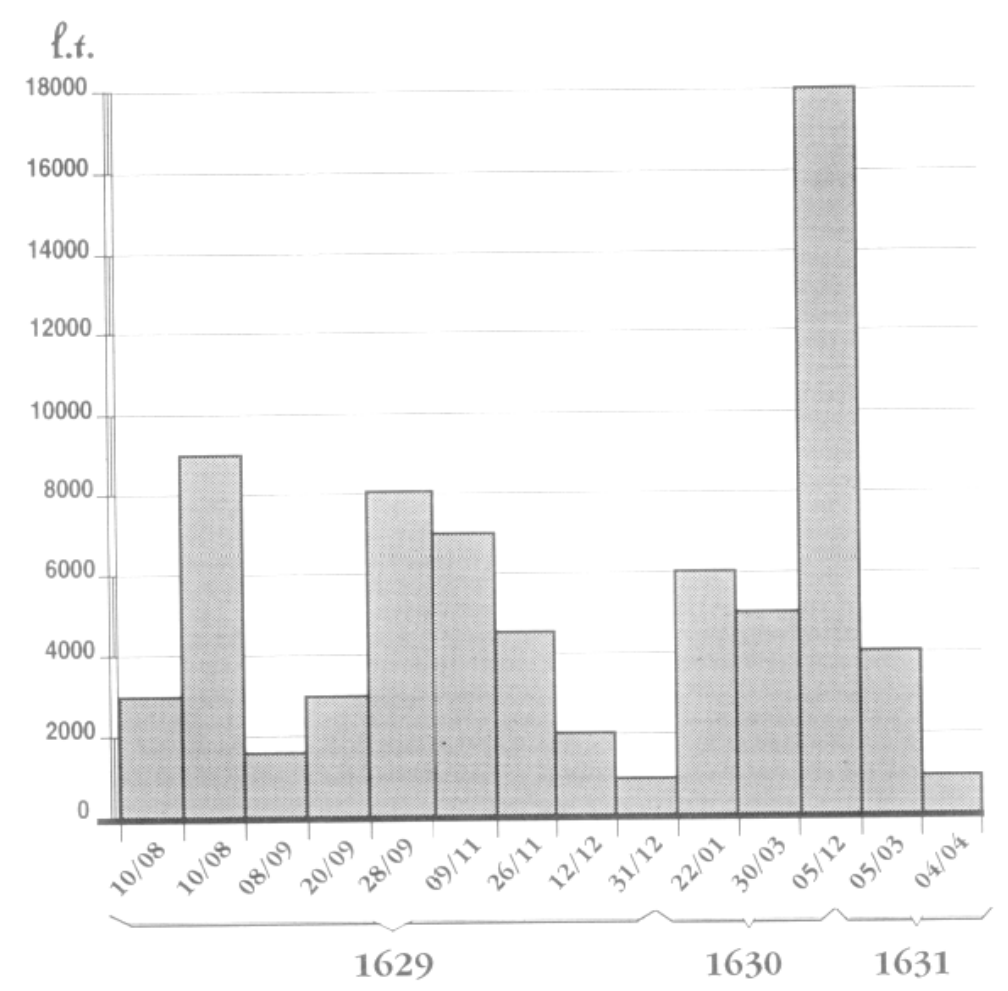

Fig. 6. - Emprunts de la ville de Bordeaux en livres tournois, 1629-1631.

des sommes prêtées lors de l'épidémie précédente s'élevaient au moins à $139751.12 \mathrm{~s} .7 \mathrm{~d} .^{61}$ soit, au denier quinze, un capital de près de 210000 livres. Le poids de la dette s'alourdit donc régulièrement, notamment en 1629-1631. Le 31 décembre 1633, le roi chargea l'intendant de Verthamon et le trésorier de France de Gourgues de vérifier les dettes de la ville, qui furent arrêtées le 10 janvier 1635 à 2481481.17 s. 1 d., soit à peu près deux à trois ans de revenus. En août 1646, le Conseil des Trente estima les dettes à un demi-million de livres, signe d'une nouvelle dégradation ${ }^{62}$, le retour rapproché des crises ne permettant pas de redresser la situation.

\section{La lutte contre le fléau}

\section{La quarantaine}

Parlement, jurade et Bureau de santé se réunirent régulièrement pour suivre l'état sanitaire, mais avant tout pour écouter les recommandations des hommes de médecine et prendre les mesures dictées par les circonstances.

61. A.C.B., CC 33.

62. A.C.B., BB 44. Aux emprunts dus aux peste s'en ajoutaient d'autres, contractés pour l'entretien des remparts ou les procès. 
Il s'agissait dans un premier temps de protéger la ville de la contagion qui menaçait, pour éviter que le mal ne l'investisse. Sur les conseils du corps médical, dès le printemps 1629, il fut décidé d'entraver la libre circulation des marchandises et notamment des tissus. Des ordres similaires furent réitérés au cours de l'été. Le Bureau ordonna qu' « aucune draperie ny cardes estoffes venans de Lion Tholouse et autres lieux suspectz n'entrera[it] en la présente ville $^{6.3} \gg$. Pour éviter l'entrée des marchandises et des individus suspects - mendiants, soldats étrangers - , la ville put $s$ 'abriter derrière les remparts qui la ceinturaient, tant du côté de la terre que de la rivière. La vigilance fut immédiatement renforcée aux portes, une dizaine, qui permettaient les liaisons avec l'extérieur. Certaines furent même fermées pour limiter la charge des archers du guet : en août 1629, la jurade fit fermer les portes Saint-Germain, SainteEulalie, Sainte-Croix, Portanets et des Paux $^{64}$; la porte Dauphine fut bloquée au cours de l'été 1630 et le 16 décembre suivant une ordonnance fixa à dix le nombre de portes ouvertes ${ }^{65}$. En juillet 1643, à nouveau, le parlement demanda aux jurats de faire garder les portes pour protéger la cité de la maladie qui ravageait alors le Limousin. Il fallut multiplier les recommandations aux gardes, exhorter les officiers, voire menacer d'amendes pour que la surveillance ne soit pas relâchée un instant. On imposa les mêmes obligations aux bourgeois, du moins à ceux qui étaient restés. En 1629, des bourgeois armés d'épées furent affectés à chaque porte de l'ouverture à la fermeture ${ }^{66}$. Faute de moyens, on dut réduire leur nombre à deux en décembre 1631, en les menaçant de les faire garder à leurs frais s'ils ne s'acquittaient pas de leur mission ${ }^{67}$. Tout mouvement de population n'était pas interdit, mais ceux qui désiraient pénétrer en ville étaient tenus d'exhiber un billet de santé délivré par les autorités de leur lieu d'origine. Cet usage, déjà mentionné cn France à la fin du XVe siècle, ne s'était généralisé qu'à la fin du XVI et au début du XVII ${ }^{\mathrm{e}}{ }^{8}$. Se protéger signifie également expulser tous ceux que l'on soupçonne de véhiculer la maladie, notamment les vagabonds. Le règlement du 25 juin 1588 ordonnait aux gens sans aveu « de vuider et se retirer en leurs diocèses [...] sous peine du fouët ${ }^{69}$ ». En 1605, à la requête du président Despaignet, il fut enjoint aux mendiants de quitter les lieux dans les vingt-quatre heures ${ }^{70}$. Les mêmes décisions furent prises en 1629-1631, en juillet 1636 et en mars 1645.

La ville connaît donc la quarantaine, elle s'isole, ou cherche à s'isoler, de l'extérieur. L'aire d'intervention du Bureau de la santé débordait largement les

63. A.C.B., GG 1214. Les produits alimentaires toulousains furent aussi prohibés (CC 27).

64. A.C.B., BB 32.

65. A.C.B., BB 33. Portes de Chapeau-Rouge, Caillau, Pont-Saint-Jean, La Grave, Salinières, des Paux sur la rivière, Saint-Julien, Sainte-Eulalie, Saint-Germain, Dauphine vers les Landes.

66. A.C.B., GG 1214.

67. A.C.B., BB 34.

68. J.-N. BIRABEN, op. cit., p. 88-90.

69. Anciens et nouveaux statuts..., op. cit.. p. 135.

70. A.C.B., GG 1212. 
limites des murailles et des faubourgs car la juridiction de la jurade s'étendait sur une vaste banlieue de part et d'autre de la Garonne. Cette banlieue fut donc soumise à une surveillance très étroite : recherche de marchandises prohibées ${ }^{71}$, interdiction aux habitants de Villenave, Yvrac et Eysines d'entrer dans la ville, recommandation au chapitre de Saint-Seurin de renvoyer les pauvres mendiants réfugiés dans la paroisse. Outre la protection contre la menace que la banlieue pouvait faire courir à la cité, on s'efforça d'y endiguer le mal en remettant de l'argent aux curés pour leurs pestiférés, on veilla à l'approvisionnement des habitants et on fit conduire des malades dans les hôpitaux ${ }^{72}$. La surveillance des jurats s'exerça même, sans grand succès, à l'extérieur de la ban lieue puisqu'à l'automne 1628 la caisse de la ville rétribua Joseph Cabannes, jurat de Langon, pour établir un poste de garde sur la Garonne. En 1629, un bourgeois fut même envoyé enquêter à Langoiran. La ville réaffirmait à l'occasion son autorité sur le plat pays ${ }^{73}$.

Mais la quarantaine fut aussi organisée à l'intérieur, contre ceux que l'on ne pouvait renvoyer chez eux. Les maisons où des cas de contagion avaient été constatés par les médecins ou les chirurgiens étaient fermées. Plusieurs possibilités s'offraient alors : rester sur place, aller à la campagne ou à l'hôpital. Les gens de condition, membres d'une « honeste famille», pouvaient faire leur quarantaine dans leur « bourdieu ». Le jurat Lavau put ainsi visiter régulièrement ses proches dans son domaine des Graves et ne fut peut-être pas étranger au décès de sa femme en 1629. Si le mal n'était pas prouvé, ils pouvaient rester chez eux sans être surveillés. On les prévenait avec des égards ${ }^{74}$. Mais les "personnes de vile condition » étaient confiées à une garde jusqu'à l'apparition de la maladie. Une fois le mal avéré, les occupants pouvaient être enfermés, leur porte scellée par la jurade et une croix peinte sur leur porte pour prévenir les voisins. Les gens ainsi cloîtrés, « contenus », étaient nourris par la ville pendant leur quarantaine, ce qui n'évitait pas les contraventions. Sous le contrôle plus ou moins strict des dizeniers ${ }^{75}$, les clés étaient confiées aux voisins, qui manifestaient parfois plus que des réticences ${ }^{76}$ ou qui montraient beaucoup de négligence, en ouvrant les portes pour apporter la nourriture. Aussi en août 1631 est-il décidé que les vivres, enfermés dans des paniers, seraient passés par les fenêtres ${ }^{77}$. Quand plusieurs maisons étaient frappées dans la même rue, les autorités prirent même la décision de faire murer les

71. À Léognan, Cestas, Gradignan, Cadaujac, Caudeyran, Bègles, Port-Neuf et dans les palus. A.C.B., CC 27.

72. À Léognan, Mérignac, Talence. A.C.B., GG 1214.

73. A.C.B., CC $27, \mathrm{BB} 32$.

74. Le jurat Dumalle en personne se rendit au domicile du conseiller du roi de Queyssac pour le « prier » de se cloîtrer chez lui. A.C.B., BB 33.

75. A.C.B., BB 33.

76. Information judiciaire en juillet 1631 contre «plusieurs personnes [qui] usent de beaucoup d'insolances lorsqu'il leur est présanté les clés $[\ldots]$ pour assister ceux quy demeurent enfermés $\gg$. A.C.B., GG 1214.

77. A.C.B., BB 34. 
deux extrémités de celle-ci pour éviter tout contact avec le reste du quartier ${ }^{78}$. Ceux qui ne déclaraient pas la maladie ou qui sortaient de leur maison étaient frappés de lourdes amendes et mis au pilori pour l'exemple. Une veuve fut même condamnée en 1632 à être "pandue et estranglée à une potance ${ }^{79}$ » dressée devant la porte Saint-Julien.

\section{Soins et hygiène}

La plupart des malades étaient toutefois envoyés dans les hôpitaux de la peste, situés à l'extérieur des remparts, l'hôpital Saint-André n'étant pas chargé de recueillir des pestiférés même si quelques cas y furent découverts. L'enclos d'Arnaud Guiraud était situé près de la grande porte Sainte-Croix, et l'hôpital de Limes ou de l'Enquesteur, près du moulin de Sainte-Croix à Paludate ${ }^{80}$. Ces deux hôpitaux avaient été établis lors de l'épidémie de 1585-1586. Le

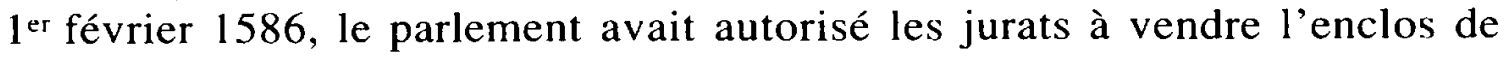
Bouglon aux fins d'acquérir pour 750 écus le bourdieu du défunt Limes dans le but d'y établir un hôpital de la peste. Le 9 octobre suivant, la jurade avait acheté à Nicolas Dupuy pour 1200 écus le bourdieu d'Arnaud Guirault, composé de «maison, grange, cave, chay, appends, jardin et puys, le tout entouré de murailles $^{81} \gg$. Les deux établissements servirent dès 1604 et lors des crises ultérieures: Arnaud Guiraud pour les soins et une quarantaine, Limes pour les convalescents. L'état lacunaire des registres ne permet pas de suivre avec précision l'évolution des effectifs. Les rapports de Vrignon témoignent en tout cas d'une dégradation de la situation en 1629 . On comptait 108 malades au début de l'été, 152 le 20 septembre, 520 fin novembre, et le 20 décembre 300 malades à Arnaud Guiraud et 240 à l'Enquesteur. Après l'accalmie des premiers mois de 1630 , les effectifs gonflent à nouveau avec 400 pensionnaires au moins à Arnaud Guiraud en septembre. L'usage de cabanes en bois, appelées loges ou huttes selon les provinces, s'était développé au cours du siècle ${ }^{82}$. Compte tenu de l'afflux des malades, les autorités en firent construire dès l'été 1629 près de l'hôpital, le long des remparts, pour héberger des familles en quarantaine car l'on séparait, dans la mesure du possible, les malades contagieux des gens encore sains ${ }^{83}$. Les personnes hospitalisées pouvaient entrer avec leurs

78. Ainsi les rues Clare et des Étuves en 1629. A.C.B., GG 1214, CC 27.

79. A.D.G., cour des jurats, 12 B 4 ( 3 février 1632). Autres sentences, moins lourdes, les 20 septembre 1629, 13 août 1631,14 février 1632 (12 B 4); les 22 septembre et $1^{\text {er }}$ décembre 1646 (12 B $6)$.

80. L'enclos Guiraud fut démoli sous le Premier Empire et remplacé par le dépôt de mendicité, actuellement lycée G.-Eiffel. Limes fut remplacé par l'hôpital des Enfants abandonnés ou de la Manufacture, remplacé au XIX' siècle par les chais Descas face à l'actuel pont Saint-Jean.

81. A.C.B., GG 1200.

82. À Montpellier, Digne, Sarlat, Albi, Foix ou Dieppe en 1629-1631. J.-N. Biraben, op. cit., p. $170-171$.

83. A.C.B., GG 1214, 6 décembre 1629. 
neubles et leur linge à condition de les faire désinfecter. Le séjour à l'hôpital stait avant tout une mise en quarantaine, car les soins prodigués par médecins zt chirurgiens n'étaient pas appropriés à la maladie. La ville versait des gages ślevés à ceux qui acceptaient de travailler au milieu des pestiférés et le titre de naître chirurgien était accordé sans examen à ceux qui se dévouaient ${ }^{84}$. La mission restait périlleuse pour le corps médical et plusieurs de ses membres y laissèrent leur vie ${ }^{85}$, même les médecins-miracle comme ce " personnage du royaume d'Alger, nommé Jean Baptiste... [qui] avoit fait en son temps des cures rares et extraordinaires ${ }^{86} \gg$. Il mourut au printemps 1605 , peu de temps après son entrée à l'hôpital. Soins médicaux, drogues fournies par les apothicaires, pilules préservatives, eaux cordiales ou thériaquales, onguents, cataplasmes de racines d'althaea, de Tapsus harbatus, et autres mixtures au vin blanc n'empêchèrent pas les plus fragiles de succomber ${ }^{87}$. Les autres, une fois leur quarantaine achevée, sortaient de l'hôpital de Limes, en présence des jurats, et rentraient chez eux, où les propriétaires étaient tenus de les accepter en vertu d'une décision du Bureau de santé de $1605^{88}$. L'épidémie enrayée, les jurats faisaient nettoyer les hôpitaux, rouvrir les écoles, et la vie reprenait son cours normal.

Autre préoccupation des jurats, assainir la ville. L'épidémie est associée, non sans raison, compte tenu du rôle des rats, à la saleté des rues et aux immondices. L'hygiène publique devient la préoccupation de tous les instants. Le 3 avril 1605 , un arrêt du parlement homologue un règlement de la jurade sur le nettoiement des rues. Le 31 juillet suivant, les jurats remettent en vigueur une ordonnance du 5 avril 1603 obligeant les habitants à nettoyer leur devant de maison $^{89}$. Cette ordonnance fut remise en vigueur dès le 21 mars 1629, au moment où la ville signait un accord avec Antoine Troubat, juge des fangas, pour assurer le nettoiement de la ville. Le parlement donna son accord et ordonna la nomination, dans chaque jurade, de trois bourgeois chargés de faire obéir les récalcitrants ${ }^{90}$. Des recommandations identiques furent réitérées les 20 novembre 1635,19 juillet 1636 et 16 mars 1645 . Il fut défendu de nourrir les pigeons, chiens, chats et lapins, de faire fondre de la graisse et du suif à l'intérieur de la ville ${ }^{91}$. Pour laver le linge des particuliers, certains lavoirs furen1 affectés aux pestiférés. En vertu des recommandations du corps médical, les maisons fermées durent être lessivées, nettoyées avec du vinaigre, blanchies e1

84. Des faveurs identiques furent accordées à Caen en 1605 ou à Lyon en 1628 . J.-N. BIRABEN op. cit., p. 133.

85. David Laporte, entré le 18 septembre 1629, était mort avant le 3 octobre. A.C.B., BB 32.

86. Chronique bordelaise, p. 122. En 1645, les jurats s'intéressèrent à un chirurgien de Turenne réputé pour ses remèdes, mais il ne semble pas qu'il soit venu à Bordeaux. A.C.B., BB 43.

87. G. PÉRY, op. cit., p. 13.

88. A.C.B., GG 1212.

89. Inventaire sommaire des registres de la jurade, 1901, vol. 2, p. 292.

90. A.C.B., BB 32.

91 A.C.B.. GG 1214. 
parfumées par les soins de cinq ou six parfumeurs ${ }^{92}$. Mais certains malade n'hésitèrent pas à sortir de l'hôpital clandestinement pour désinfecter leu demeure, contribuant ainsi à l'extension de l'épidémie.

\section{Nourrir les Bordelais}

L'alimentation restait un problème crucial. Compte tenu de la perturbatior des circuits économiques, de la mise en quarantaine d'une partie de la population, ce fut aux autorités qu'incomba l'approvisionnement de la cité. Dans un premier temps, la jurade fit acheter du blé et du vin pour les hôpitaux et pouı les pauvres à qui l'on distribuait des billets. Ces distributions entraînèrent des abus qu'il fallut corriger et les jurats, assistés de dizeniers, durent vérifier les listes de leur quartier et sévir, le 3 janvier 1630 , contre le responsable d'Arnaud Guiraud qui réservait «pour sa table les meilleurs vivres ${ }^{93}$ ». À partir de mai 1630, la démarche évolua car le prix des céréales augmenta dans des proportions importantes (figure 4). On procéda alors à des visites de greniers chez les particuliers et les boulangers pour connaître les disponibilités et éviter l'accaparement ${ }^{94}$. Le 31 juillet 1630 , le Bureau de la santé recommanda aux habitants de faire des provisions pour six mois ou un an, alors que le boisseau de froment se vendait déjà 10 livres. Comme lors de la crise de 1604-160595, le parlement et la jurade interdirent l'exportation des grains, ce qui contraria bien des intérêts particuliers et suscita des dissensions au sein du corps de ville. Le 24 novembre 1631, les jurats Ducournaud et Dumalle apostrophèrent leur collègue Jean-Paul Achard qui avait fait charger en septembre une quarantaine de boisseaux de froment pour ses métayers de Mauconseil, niant l'octroi d'une permission verbale à leur collègue. La jurade dut se fournir en grains pour éviter la misère et les émotions populaires. On sollicita le soutien de Monsieur et, le 3 septembre 1630, Louis XIII permit de se fournir en Bretagne, Normandie et Picardie. Des lettres furent envoyées au duc d'Épernon, gouverneur de la province, au parlement de Rennes, et pour accélérer la décision le jurat de Bétoulaud et le bourgeois Hugla furent envoyés en députation. De retour le $1^{\text {er }}$ avril 1631, ils pouvaient annoncer l'accord donné le 3 janvier par le parlement breton pour 1000 tonneaux ${ }^{96}$. Des navires chargés de blé arrivèrent dans le port, et malgré quelques tensions avec certains négociants, des distributions furent organisées. Le prix des blés s'effondra entre mai et juillet 1631, revenant ıux niveaux antérieurs. Les démarches avaient abouti, mais après une année de vie chère au cours de laquelle la disette avait renforcé les effets néfastes de

92. On utilisait à cet effet de l'encens ou des bois odoriférants comme pins, sapins ou lauriers.
93. A.C.B., GG 1214 .

94. En juin 1630, mai et août 1631 au moins. A.C.B., BB 32, 33, 34.

95. Chronique bordelaise, p. 121.

96. A.C.B., BB 33. 
l'épidémie. En 1645, la cherté était apparue avant la peste, malgré les décisions des autorités - visite des greniers, constitution de réserves -, et la ville ne semble pas avoir connu un déficit alimentaire aussi grave qu'en 1630 .

\section{Les secours de la religion}

Dans une société chrétienne, les malheurs des hommes doivent avoir une justification. Si le Seigneur n'avait pas protégé la population, c'était en raison de son inconduite. «Ce fléau devoit venir du ciel pour les péchez du peuple » indique la Chronique bourdeloise; il fallait se réconcilier avec Dieu, et les règlements municipaux commençaient par des intercessions ${ }^{97}$. Jurats et parlementaires participèrent régulièrement à des offices, à des processions, firent dresser des feux dans les rues pour expier « la faute de la ville », firent dire des neuvaines pour le roi saint Louis ${ }^{98}$. À la différence du clergé séculier, le clergé régulier compta de nombreuses victimes, jésuites, capucins, augustins ou récollets rivalisant de courage pour réconforter les malades, confesser les mourants, visiter les hôpitaux ${ }^{99}$. Les capucins Simon de Rodez et Polycarpe de Marsiac moururent en odeur de sainteté lors de la peste de $1604-1606^{100}$. Le cardinal de Sourdis donna l'exemple en visitant les quartiers les plus touchés et en distribuant l'eucharistie ${ }^{101}$. On rechercha avant tout la protection de la Vierge Marie pour qu'elle intercède en faveur des habitants affligés. Le 23 juin 1605 , Alfonso d'Ornano et les jurats s'engagèrent à offrir un exemplaire en argent des armoiries de la ville à Notre-Dame de Lorette ${ }^{102}$. Les jurats firent dire des litanies, des messes pour l'Annonciation et la Visitation, organisèrent une procession à Saint-Seurin ${ }^{103}$. Tous les témoignages concordent sur l'affluence dans les églises lors des épidémies, notamment le 15 août, et dans les chapelles des hôpitaux ${ }^{104}$. À la fin de l'épidémie, les autorités organisaient des processions générales pour remercier Dieu d'avoir détourné sa colère de Bordeaux.

Tragique réalité de cette première moitié du Grand Siècle, la peste a donc frappé Bordeaux à plusieurs reprises sans que les autorités aient pu enrayer la

97. Anciens et nouveaux statuts..., op. cit., p. 128.

98. A.C.B., GG 1214.

99. B. PEYRous, La Réforme catholique à Bordeaux (1600-1719), Bordeaux, 1995, p. 60-61.

100. A.C.B., ms 583, Memorabilia .... op. cit., p. 345-347.

101. L.-W. Ravenez, Histoire du cardinal de Sourdis, Bordeaux, 1867, p. 95.

102. Cas similaires (lampes, statues, navires) à Thiers en 1629, à Dieppe en 1630, à Amiens en 1633. J.-N. BiRABEN, op. cit., p. 273-274.

103. Chronique bordelaise, p. 124-125.

104. A.C.B., GG 1214, BB 32. 
progression du mal. La médecine de l'époque était en effet impuissante contre la maladie, dont les vecteurs ne furent découverts qu'à l'époque contemporaine. Des causes majeures de propagation : contact avec les malades, manque d'hygiène, déplacement des personnes et de certaines marchandises, étaient certes reconnues de manière empirique, mais cela ne pouvait qu'aider à circonscrire ou ralentir l'épidémie, pas à la juguler. Or si la peste semble s'interrompre d'elle-même et reprendre à sa guise, c'est en partie la conséquence d'un manque de moyens des autorités qui ne pouvaient assurer le contrôle des mesures sanitaires qu'elles prenaient. Les gens négligeaient de nettoyer les rues, achetaient des vêtements d'originc doutcuse, des malades sortaient des hôpitaux et entraient en ville sans trop de difficultés. Les autorités elles-mêmes donnaient le mauvais exemple : les jurats ne cessèrent d'aller et venir entre leur hôtel urbain et leur bourdieu campagnard, en Blayais ou en Sauternais, se rendirent en députation à Cadillac, en Bretagne, en prenant le risque de propager la maladie ; des religieux, si dévoués par ailleurs, ne respectèrent pas toujours les consignes de quarantaine après avoir servi dans les hôpitaux. On comprend ainsi la réapparition récurrente de la contagion lors de la Fronde et au début du règne personnel de Louis XIV, les autorités bordelaises utilisant les mêmes méthodes que le reste du royaume. Les dernières mesures préventives furent prises lors de la peste de Marseille en 1720-1721, et Bordeaux vécut ensuite, comme le reste du royaume, sans la hantise de ce mal séculaire ${ }^{105}$. 